\title{
SQUAMANITA UMBONATA (FUNGI, AGARICALES, TRICHOLOMATACEAE), PRIMER REGISTRO EN MÉXICO
}

\author{
Alonso Cortés-Pérez ${ }^{1}$, Gastón GuZmán ${ }^{1,2}$ y Florencia Ramírez-GuilléN ${ }^{1}$ \\ ${ }^{1}$ Instituto de Ecología, A.C., Apdo. postal 63, \\ 91070 Xalapa, Veracruz, México. \\ ${ }^{2}$ Autor para la correspondencia: gaston.guzman@inecol.mx
}

\section{RESUMEN}

Se registra por primera vez para la micobiota mexicana el género Squamanita con $S$. umbonata, procedente de un bosque de pino-encino en el estado de Veracruz. Esta especie ha sido citada de Estados Unidos de América (Pennsylvania y Massachusetts), China y Japón. El material estudiado coincide bien con el holotipo de S. umbonata y con otro espécimen del mismo país depositado en el herbario NY, estudiado por Bas.

Palabras clave: hongos, micoparásito, nuevo registro, taxonomía.

\section{ABSTRACT}

The genus Squamanita is reported for the first time in Mexico, with S. umbonata from a pine-oak forest in the state of Veracruz. This mushroom has been cited from the United States of America (Pennsylvania, Massachusetts), China and Japan. The Mexican specimen agrees well with the holotype of $S$. umbonata, and with a specimen from the United States of America deposited in the herbarium NY studied by Bas.

Key words: mushrooms, mycoparasite, new record, taxonomy.

\section{INTRODUCCIÓN}

El género Squamanita cuenta con 10 especies (Kirk et al., 2008) y se caracteriza porque los basidiomas se originan de un cecidiocarpo (hipertrofia en la base del 
basidioma provocada por otro hongo), del cual se desarrollan uno o más basidiomas. Se distingue también porque presenta escamas flocosas en el estípite y en el píleo y por sus basidiosporas amiloides o inamiloides. Parasita a Agaricales como Galerina, Hebeloma, Inocybe y Cystoderma, entre otros (Bas, 1965; Redhead et al., 1994; Bas y Thoen, 1998; Mondiet et al. 2007; Matheny y Griffith, 2010). Es un género no muy común, pero se conoce de América del Norte y del Sur (Sumstine, 1914; Smith y Singer, 1948; Redhead et al., 1994; Bas y Laessoe, 1999), de Europa (Bas, 1965; Mondiet et al., 2007), África (Bas y Thoen, 1998) y de Asia (Harmaja, 1988; Wang y Yang, 2004). Ha sido confundido o interpretado como Vaginata (Sumstine, 1914) y Cystoderma (Smith y Singer, 1948). Sin embargo, no se había encontrado en México.

\section{MATERIALES Y MÉTODOS}

El material estudiado está depositado en la Colección de Hongos del Instituto de Ecología de Xalapa (XAL). Su análisis microscópico se basó en preparaciones elaboradas con cortes a navaja del basidioma y montadas en solución de $\mathrm{KOH}$ a 5\% o rojo Congo, previamente rehidratados con alcohol de $70^{\circ}$, o con azul de algodón, azul de cresil o solución de Melzer, dependiendo de las características anatómicas observadas. Se estudiaron y midieron por lo menos 30 de las estructuras con valor taxonómico de cada uno de los basidiomas (el mexicano, el holotipo y el otro de NY), las cuales se dibujaron a escala. En las medidas del basidioma presentadas se da una del píleo y dos del estípite (longitud y grosor), debido a que solo se examinó un basidioma en la única colecta mexicana encontrada. Las medidas entre paréntesis en las estructuras microscópicas indican extremos poco frecuentes.

\section{RESULTADOS}

Squamanita umbonata (Sumst.) Bas, Persoonia 3: 334. 1965. Figs. 1-15.

= Vaginata umbonata Sumst., Mycologia 6: 35. 1914.

= Armillaria umbonata (Sumst.) Murrill, North. Amer. Fl. 10: 38.1914.

Píleo de $34 \mathrm{~mm}$ de ancho, cónico a umbonado, liso en el centro a densamente flocoso hacia el margen, superficie algo lubricosa, de color café amarillento, con el centro más obscuro a blanquecino hacia el margen, las fibrillas del margen son blanquecinas o de color amarillo paja. Contexto carnoso, blanquecino. Láminas ad- 

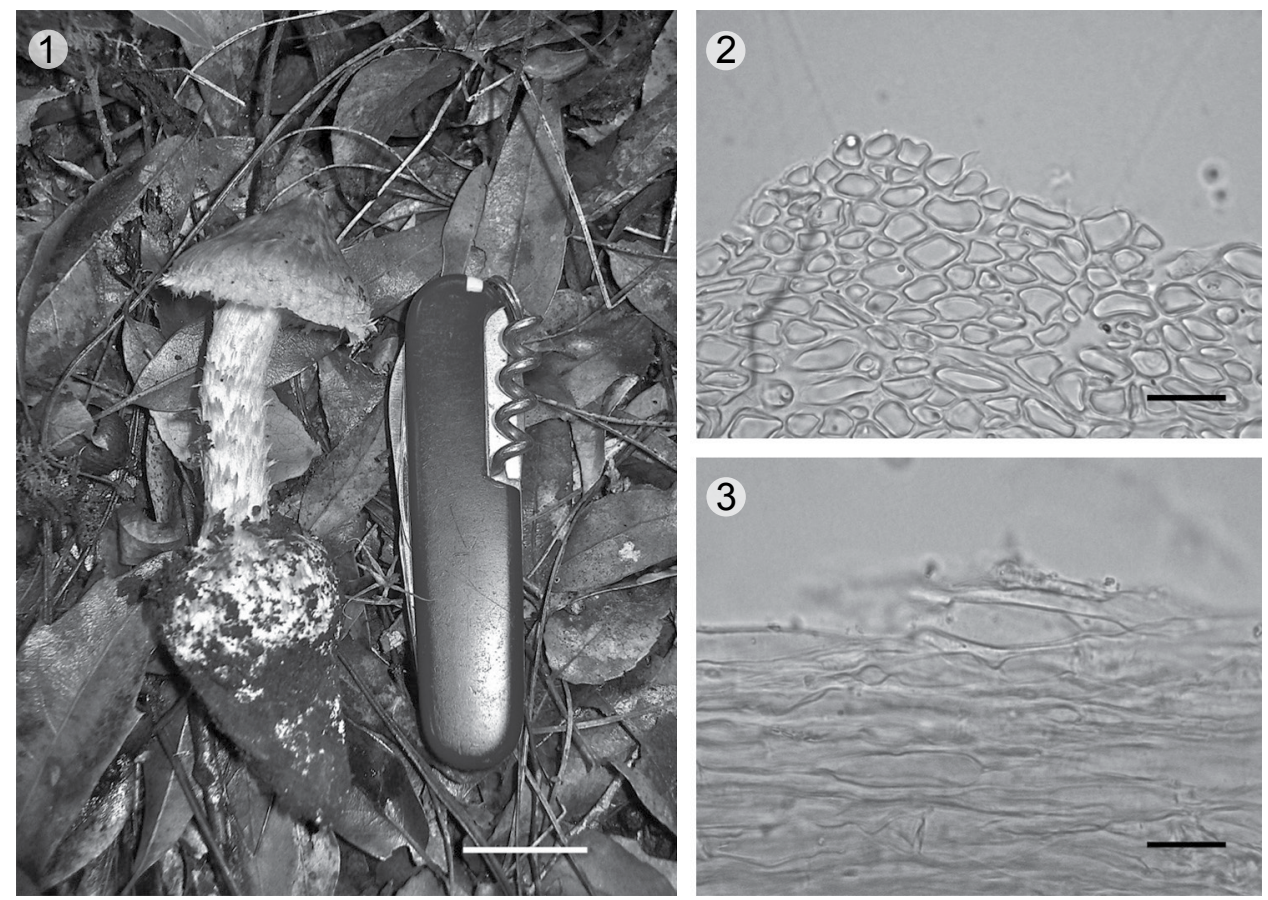

Figs. 1-3: Squamanita umbonata. 1: basidioma, nótense las escamas flocosas del borde del píleo y en el estípite; 2: pileipelis; 3: estipitipelis (1-3: Cortés-Pérez 154). Escala $1=2 \mathrm{~cm}$; Escala 2-3 $=20 \mu \mathrm{m}$.

heridas, margen entero, blanquecinas. Estípite de $44 \times 10 \mu \mathrm{m}$, fibriloso-carnoso, superficie blanquecina, flocosa-escuamulosa desde la base hasta tres terceras partes del estípite, escuamulas de color café-amarillento pálido. Cecidiocarpo de $42 \times 23$, napiforme, escamoso-flocoso arriba a más o menos liso hacia abajo, blanquecino.

Basidiosporas de (5.5-) 6-7 (-9) × (3.5-) 4-5 $\mu \mathrm{m}$, elipsoides, algunas reniformes, de pared delgada (menos de $1 \mu \mathrm{m}$ de grosor), hialinas, inamiloides, a veces rojizas pálidas con solución de Rojo Congo, no metacromáticas con azul de cresil. Basidios de (24-)(27-) 31-42 × 8-10 $\mu \mathrm{m}$, tetraspóricos, subclaviformes, hialinos. Pleurocistidios de (45-)(50-) 55-68 (-73)(-78)(-89) × (9-) 10-18 (-22) $\mu \mathrm{m}$, fusiformes a fusiforme-ventricosos, de pared delgada, algunos la presentan gruesa hacia el ápice, hialinos, a veces con incrustaciones en el ápice. Queilocistidios de (44-)(52-) 55-78 (-80)(-85) × 9-18 (-20) (-23) $\mu \mathrm{m}$, similares a los pleurocistidios. Trama himenial regular, con hifas de 2-18 $\mu \mathrm{m}$ de ancho, hialinas, a veces con incrustaciones de color amarillo pálido. Hifas oleíferas presentes. Subhimenio ramoso con hifas subglobosas, hialinas. Trama contextual ra- 


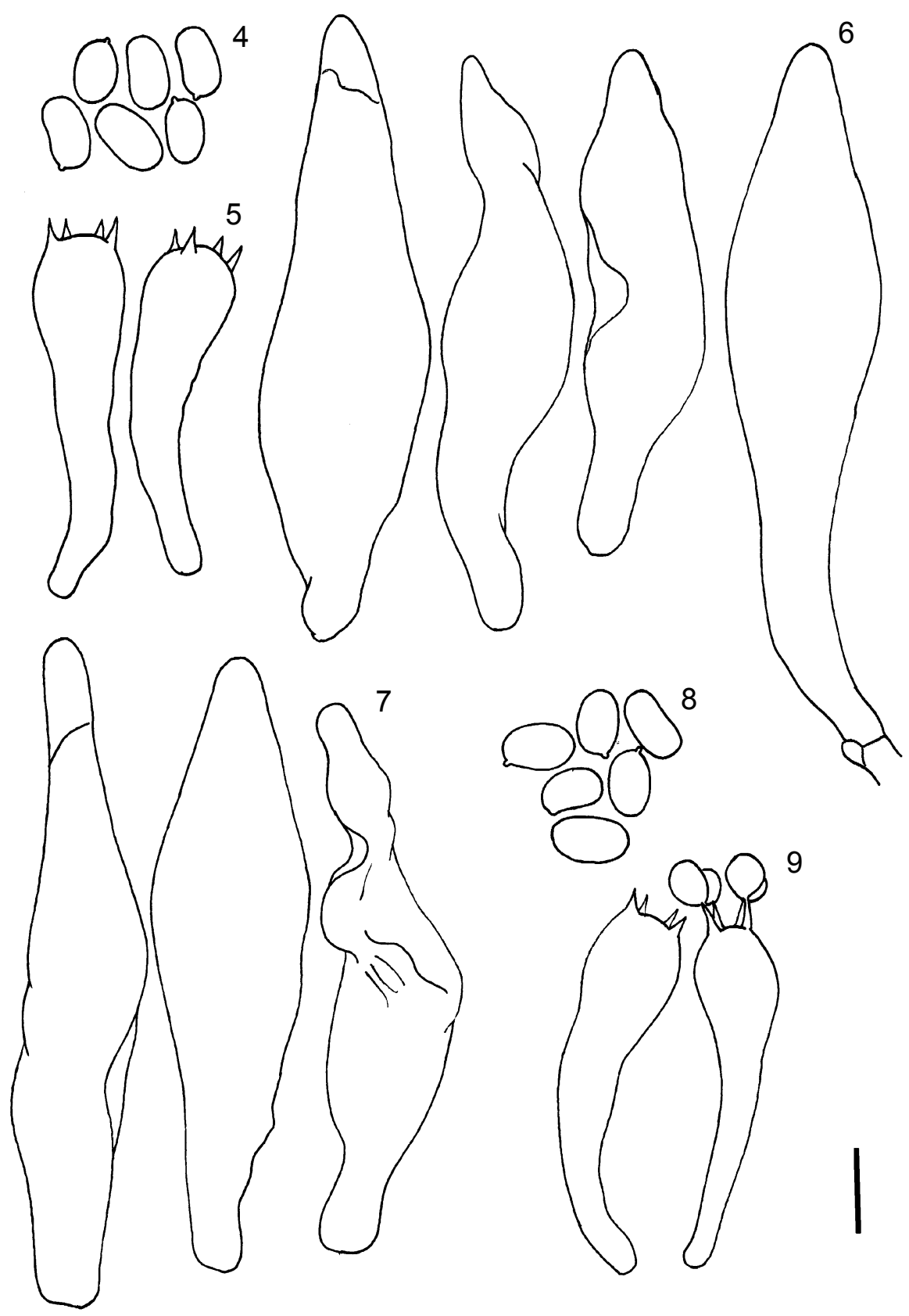

Figs. 4-9: Squamanita umbonata. 4: basidiosporas; 5: basidios; 6: pleurocistidios; 7: queilocistidios; 8: basidiosporas; 9: basidios (4-7: holotipo; 8-9: Cortés-Pérez 154). Escala $=10 \mu \mathrm{m}$. 
Cortés-Pérez et al.: Squamanita umbonata, primer registro en México
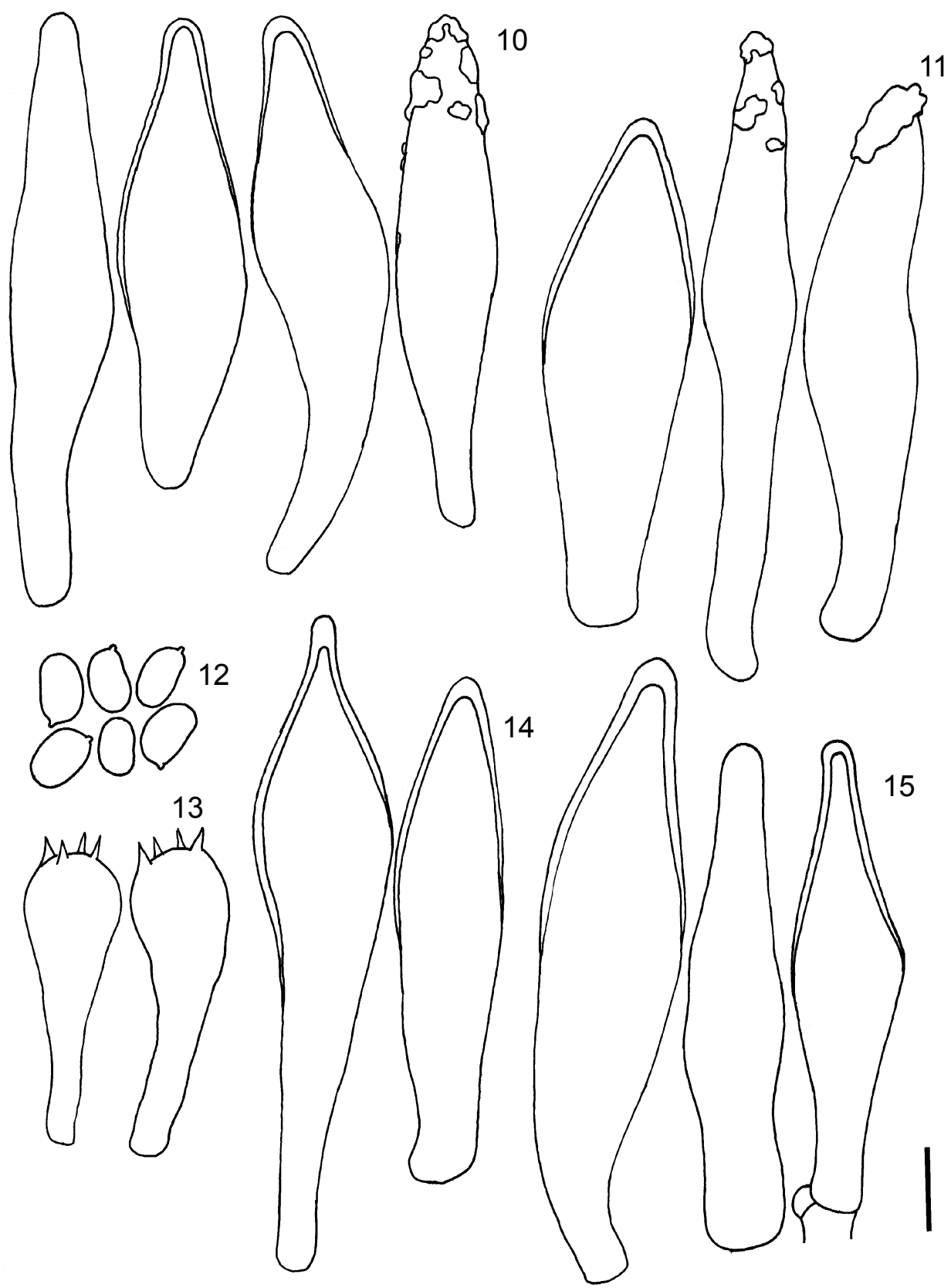

Figs. 10-15: Squamanita umbonata. 10: pleurocistidios; 11: queilocistidios; 12: basidiosporas; 13: basidios; 14: pleurocistidios; 15: queilocistidios (10-11: Cortés-Pérez 154; 12-15: Bas 3808). Escala $=10 \mu \mathrm{m}$. 
dial, hifas de 3-25 (-32) $\mu \mathrm{m}$ de ancho, de pared delgada o de $1 \mu \mathrm{m}$ de grosor, hialinas con incrustaciones amarillas. Pileipelis como un cutis, con hifas dispuestas radialmente, no diferenciadas de la trama contextual, de 3-18 $\mu \mathrm{m}$ de ancho, hialinas, con incrustaciones amarillas. Estipitipelis con hifas postradas, de (2-) 3-21 $\mu \mathrm{m}$ de ancho, de pared delgada, hialinas, con incrustaciones amarillas. Fíbulas presentes.

Material estudiado: E.U.A., Pennsylvania, Fayette Co., Ohiopyle, agosto 12, 1908, D.R. Sumstine (Holotipo, NY); Massachusetts, Mt. Toby Forest, agosto 25, 1963, C. Bas 3808 (NY); MÉXICO, Veracruz, Mpio. de Huayacocotla, lado este de Huayacocotla, septiembre 13, 2009, A. Cortés-Pérez 154 (XAL).

El material mexicano concuerda bien en sus características taxonómicas con el holotipo y con la colecta de Bas de Massachusetts. Sumstine (1914) definió esta especie por el píleo umbonado, con escamas, con estípite largo y bulboso y volva fimbriada. Más tarde, Murrill en 1914 (según Bas, 1965) consideró el hongo de Sumstine en el género Armillaria. Bas (1965) transfirió la especie a Squamanita por la presencia de un cecidiocarpo, el píleo umbonado y el estípite irregularmente escamosofibriloso. La separó de $S$. schreieri Imbach de Europa, por la ausencia de cistidios. Wang y Yang (2004) citaron cistidios de pared ligeramente gruesa o gruesa. Un taxon muy cercano es $S$. citricolor Thoen que habita en África central, el cual se diferencia por presentar píleo y estípite glabro, así como cistidios de pared gruesa (Bas y Thoen, 1998). El resto de las especies conocidas en el género no tienen cistidios (Bas, 1965). Referente al micoparasitismo en el cecidiocarpo, Redhead et al. (1994) registraron a de Squamanita sobre hospedantes tales como Amanita, Cystoderma, Galerina, Kuehneromyces y Phaeolepiota. Posteriormente Mondiet et al. (2007) hicieron ver que Squamanita odorata (Cool) Bas parasita a Hebeloma mesophaeum (Pers.) Quél.

\section{AGRADECIMIENTOS}

Los autores agradecen a Juan Lara Carmona y Manuel Hernández por su colaboración en el herbario y en actividades de cómputo, respectivamente. Se dan gracias también al curador del herbario NY por el préstamo enviado.

\section{LITERATURA CITADA}

Bas, C. 1965. The genus Squamanita. Persoonia 3: 331-359.

Bas, C. y T. Laessoe. 1999. Squamanita granulifera sp. nov. A first record of Squamanita (Agaricales) from South America. Kew Bull. 54: 811-815. 
Bas, C. y D. Thoen. 1998. Squamanita citricolor, a new species from central Africa. Persoonia 17: 135-139.

Harmaja, H. 1988. Studies on the agaric genera Singerocybe n. gen. and Squamanita. Karstenia 27: 71-75.

Kirk, P. M., P. F. Cannon, D. W. Minter y J. A. Stalpers. 2008. Ainsworth \& Bisby's dictionary of the fungi. 10a. ed. CABI. Wallingford, UK. 771 pp.

Matheny, P. B. y G. W. Griffith. 2010. Mycoparasitism between Squamanita paradoxa and Cystoderma amianthinum (Cystodermateae, Agaricales). Mycoscience 51: 456-461.

Mondiet, N., M. P. Dubois y M. A. Selosse. 2007. The enigmatic Squamanita odorata (Agaricales, Basidiomycota) is parasitic on Hebeloma mesophaeum. Mycol.Res. 111: 599-602.

Redhead, S. A., J. F. Ammirati, G. R. Walker, L. L. Norvell y M. B. Puccio. 1994. Squamanita contortipes, the Rosetta Stone of a mycoparasitic agaric genus. Can. J. Bot. 72: 18121824.

Smith, A. H. y R. Singer. 1948. Notes on the genus Cystoderma. Mycologia 40: 454-460.

Sumstine, D. R. 1914. New or interesting fungi. Mycologia 6: 32-36.

Wang, H. Ch. y Z. L. Yang. 2004. Squamanita, a new record to China. Mycosystema 23(1): 146-148. 